\title{
Community Malaria Knowledge, Experiences, Perceived Roles, and Acceptability of Community-Directed Distribution of Intermittent Preventive Therapy for Pregnancy in Rural Southeast Nigeria
}

\author{
Ijeoma Nkem Okedo-Alex, ${ }^{1,2}$ Ifeyinwa Chizoba Akamike,, ${ }^{1,2}$ Johnbosco Ifunanya Nwafor, ${ }^{3}$ \\ Chihurumnanya Alo, ${ }^{2}$ Adaoha Pearl Agu, ${ }^{1,2}$ Dejene Derseh Abateneh $\mathbb{D},{ }^{4}$ \\ and Chigozie Jesse Uneke ${ }^{1}$ \\ ${ }^{1}$ African Institute for Health Policy and Health Systems, Ebonyi State University, Abakaliki, Nigeria \\ ${ }^{2}$ Department of Community Medicine, Alex Ekwueme Federal University Teaching Hospital Abakaliki Ebonyi State, Nigeria \\ ${ }^{3}$ Department of Obstetrics and Gynaecology, Alex Ekwueme Federal University Teaching Hospital, Abakaliki, Ebonyi State, Nigeria \\ ${ }^{4}$ Kotebe Metropolitan University, Menelik II College of Medicine and Health Sciences, Department of Medical Laboratory Sciences, \\ Addis Ababa, Ethiopia
}

Correspondence should be addressed to Dejene Derseh Abateneh; dejenieh@gmail.com

Received 25 June 2021; Accepted 28 December 2021; Published 18 January 2022

Academic Editor: Eric Agola Lelo

Copyright () 2022 Ijeoma Nkem Okedo-Alex et al. This is an open access article distributed under the Creative Commons Attribution License, which permits unrestricted use, distribution, and reproduction in any medium, provided the original work is properly cited.

Background. The community plays key roles in protecting pregnant women in rural areas from malaria. This study assessed malaria experiences, knowledge, perceived roles in malaria prevention in pregnancy, and acceptability of community-directed distribution of intermittent preventive therapy (IPTp) for malaria in pregnancy in rural Southeast Nigeria. Methods. This study presents part of the baseline findings of a before-and-after study. Data was collected from 817 community members in Ebonyi State using interviewer-administered questionnaires and focus group discussions (FGDs). Data were analyzed using SPSS version 20 and thematic analysis. Results. The majority of the respondents were females $(73.8 \%)$ with a mean age of $36.08 \pm$ 15.4. Most respondents (65.2\%) had Insecticide-Treated Net (ITN) and fever in the past year (67.1\%). Malaria (88.6\%) was identified as the major health condition in the community. Majority (74.1\%) knew infected mosquito bites as the cause of malaria while $61.1 \%$ and $71.5 \%$ were definitely sure that pregnant women and children were at risk for malaria. Sleeping under ITN (54.3\%), clean environment (39.7\%), and herbal medications (26.8\%) were the main ways of malaria prevention cited. Only $18.4 \%$ of the participants rated their knowledge of IPTp as adequate, and only $9.3 \%$ knew the common drug names used for IPTp. The major perceived roles in malaria prevention in pregnancy were referral of pregnant women to the health facility, encouragement of household ITN use, and sustaining malaria-related projects. The majority of the participants (60.6\%) strongly agreed that community-directed distribution of IPTp-SP will improve the prevention of malaria in pregnancy. Most $(77.2 \%)$ considered community-directed distribution of IPTp acceptable, and $74.4 \%$ of the pregnant respondents preferred community to facility administration of IPTp. Conclusions. Malaria was recognized as a prevalent disease, but there was inadequate knowledge of malaria prevention in pregnancy notably intermittent preventive therapy. There was positive perception of roles in malaria prevention in pregnancy and high acceptability of community-directed distribution of IPTp. Community-level malaria control programs should utilize a whole-of-community approach to optimally engage and educate the community on malaria prevention in pregnancy as well as explore community distribution approach for IPTp. 


\section{Introduction}

According to the World Malaria Report 2021 (page xv), the World Health Organization (WHO) African Region continues to bear the global malaria burden, accounting for about $95 \%$ of the 241 million malaria cases worldwide and $96 \%$ of the 627000 deaths from malaria in 2020 [1]. Nigeria significantly contributes to the global burden of malaria cases as $27 \%$ of all malaria cases in 2020 occurred in Nigeria [1]. Malaria is holoendemic in Nigeria and remains a major public health problem, taking its greatest toll on children under the age of 5 and pregnant women, although it is preventable, treatable, and curable [2]. Malaria in pregnancy (MIP) is associated with varying symptoms and can be complicated with anemia, stillbirths, prematurity, and low birth weight (LBW). Malaria-associated maternal illness and low birth weight are mostly the result of Plasmodium falciparum infection and occur frequently in Nigeria [3]. The disease overstretches the nation's struggling health system with grave socio-economic consequences for both household and national levels. This is manifested in reduced gross domestic product, productivity, and high out-of-pocket expenditures on treatment and prevention expenditures [4].

The transmission of malaria in Nigeria is endemic and perennial. There are favorable climatic conditions and a higher prevalence of malaria after the end of the rainy season $[5,6]$. The predominant Anopheline vector species in Nigeria are An. gambaie sp, An. arabiensis, and An. Funestus. Malaria transmission is highest in rural communities with enabling environmental conditions for vector breeding, and this is further compounded by the fragile health system arrangements in these areas. Pregnant women and underfive children in such remote areas remain worse hit by malaria [7].

To prevent and ensure proper treatment of malaria in pregnancy, the three cardinal approaches recommended by the World Health Organization (WHO) are the use of long-lasting Insecticide-Treated Nets (ITNs), prompt diagnosis, and effective treatment of malaria infection and intermittent preventive treatment in pregnancy with suphadoxine-pyrimethamine (IPTp-SP) as part of antenatal services in areas of moderate to high transmission of $P$. falciparum [3].

At the community and household level, effective prevention and control of malaria are largely dependent on community members properly identifying malaria as a priority health issue. The community's knowledge and perceptions of malaria could influence practices that predispose or protect from malaria $[8,9]$.

The community could play key roles in protecting pregnant women from malaria by encouraging communal practices and norms such as spousal involvement, community support of antenatal attendance, ITN and IPTp use, and environmental sanitation as well as collaborating with and sustaining health programs on malaria prevention. In 2018, only $46 \%$ of Nigerian women in rural areas had at least four antenatal care (ANC) visits, and only $14 \%$ of ANC users initiated these visits in the first trimester [6]. This conspicuously falls short of the minimum eight visits and early
ANC initiation advocated by the WHO [10]. Although Nigeria reflected the WHO recommendations of eight contact visits in its 2017 ANC orientation package for health care providers [11], this most recent national survey on ANC utilization was based on attending at least four ANC visits. Suboptimal antenatal care utilization and by extension, IPTp uptake remains a burgeoning concern in the prevention of malaria in pregnancy in Nigeria [6].

Community approaches have been increasingly recognized because they improve community acceptability and ownership as the structures used are part of the community in addition to the existing community solidarity. They also obviate the creation of new structures for health interventions $[12,13]$. Studies have shown that community-based programs can substantially increase effective access to IPTp and other types of malaria prevention in addition to increasing access to antenatal care and other formal health care access in general [14-16]. Such community-level programs include community-directed distribution of preventive malaria commodities such as IPTp-SP by trained community distributors, community sensitization, and community case management of malaria by community health workers $[14,17,18]$. Although such community-level interventions have been shown to be contextually relevant and effective in resource-constrained settings $[14,19,20]$, very few studies have assessed their acceptability by community members as acceptability can be an important precursor of sustainability. The aim of this study was to assess community malaria experiences, knowledge, perceived roles in prevention of malaria in pregnancy, and acceptability of communitydirected distribution of intermittent preventive therapy for malaria in pregnancy in rural Southeast Nigeria. The findings are part of the baseline findings of an intervention study to implement community-directed distribution of intermittent preventive therapy for the prevention of malaria in pregnancy [19].

\section{Materials and Methods}

2.1. Study Area. This study was conducted in three communities (Okuzzu-Ukawu, Isinkwo, and Abomege) located in Ukaba Development Centre of Onicha local government area (LGA) in Ebonyi State, Southeast Nigeria. Ebonyi State has three senatorial zones and thirteen LGAs. According to the 2006 population and housing census, the population of Ebonyi state is approximately 2,176,947 with a landmass of 5,935 square kilometers. Infants (children $<1$ year old) make up $4 \%$ of the population, children under five $20 \%$, and women of childbearing age $22 \%$ of the population [21]. The people of Ukawu are mostly Ibos, the dominant tribe of southeast geopolitical zone of Nigeria, and their major occupations include farming and trading.

Using the most recent national census (1991) definition, a rural area is defined as a settlement with $<20000$ inhabitants [22]. The study area had $<20000$ inhabitants. Nigeria has also been shown to be dominantly rural [23].

Ebiriogu community located in Okuzzu-Ukwau political ward was the intervention community for the communitydirected IPTp distribution project. More details about the 
intervention have been published elsewhere [19]. The community has three settlements and one primary health center (PHC) which is the major source of orthodox health care services in the community. However, people of the community also access health services in the PHCs located in the other political wards as well as from traditional healers.

2.2. Study Population. Data was collected from adult men and women in the settlements in Ebirogu community. Only those who resided in the community and gave informed consent were surveyed. To ensure adequate participation of women of childbearing age who are the primary targets of IPTp, nursing and pregnant mothers in the community were encouraged to participate in the study. Also, those attending immunization and antenatal clinics in the high patronage PHCs in Ukawu Development center were consecutively recruited into the study (for the interviews) over a threeweek period. Opinion leaders and pregnant/nursing mothers in the community participated in the focus group discussions.

2.3. Study Design. The study utilized a descriptive crosssectional study design using a mixed method approach comprising semistructured interviews and focus group discussions.

2.4. Sample Size Determination and Sampling. The WINPEPI statistical software for epidemiologists was used to calculate the minimum sample size using the formula for a single proportion $[24,25]$. We assumed that at least $50 \%$ of the respondents would consider community-directed distribution of IPTp acceptable. A minimum sample size of 385 was calculated and recalculated to 427 after adjusting for a nonresponse rate of $10 \%$. To improve the robustness (representativeness and precision) of the study, the sample size was doubled to 854. Eight hundred and seventeen participants responded to the questionnaires to give a response rate of $95.7 \%$. Using the settlements within the community as clusters, two clusters were selected by balloting and all households in each cluster sampled for eligible participants in the community. All eligible participants who provided written informed consent were surveyed.

2.5. Data Collection Methods. Semistructured intervieweradministered questionnaires and focus group discussions (FGD) were used for data collection. Data was collected before the onset of the community-directed distribution of IPTp as part of the baseline data in May-June 2019. The questionnaire had three sections, and the first section collected information on socio-demographic and malariarelated characteristics, burden of malaria and knowledge of prevention using IPTp and community ownership, and acceptability of community-directed distribution of IPTp. The second section explored the burden of malaria, knowledge of the causes, at-risk-populations, and intermittent preventive therapy for malaria in pregnancy. The third section of the questionnaire explored perceptions on community roles, ownership, and acceptability of community-directed distribution of IPTp for prevention of malaria in pregnancy. The questionnaire was adapted from domains on malaria explored in the National Malaria Indicator Survey [2]. It was pretested among adult community members in another rural community who are not part of the study population in order to improve the clarity and arrangement of the questions. The questionnaires were administered by trained graduate research assistants.

The FGDs were used to further explore and understand community perceptions on the prevalent health challenges and burden of malaria in the community, community roles in preventing malaria in pregnancy, and acceptability of community-directed distribution of IPTp for prevention of malaria in pregnancy.

Two FGDs were held using a focus group discussion guide developed by the researchers. The FGD guide had a total of eight questions. Each FGD had 10-12 participants and lasted for about 40 minutes. One of the FGDs was held with nursing/pregnant mothers while the other FGD was held with opinion leaders in the community. With the assistance of the officer-in-charge of the PHC in Ebiriogu who acted as a link person, we purposively selected male and female opinion leaders because the project is aimed at ascertaining major health issues in the community and community support towards the project. They were encouraged to air their views freely because adequate understanding of the subject under study required that participants freely articulate and vocalize their views. The participants were approached face-to-face, and the purpose of the research was clearly explained to them. They were at liberty to opt out at will, but all agreed to participate. We used an electronic recorder to record the discussions and obtained permission to record from the respondents before commencing the FGDs. The officer-in-charge of the PHC in Ebiriogu also facilitated the selection of nursing and pregnant mothers in the community on the basis of willingness to participate in the FGD.

The principal researcher (female) moderated the discussions, and she was assisted by a research assistant who acted as the note-taker to record the significant inputs made by the discussants including nonverbal reactions. To ensure that the opinions of the FGD participants were appropriately captured, the moderator intermittently summarized the responses and sought their feedbacks on the accuracy of the summaries.

\subsection{Data Management}

2.6.1. Measurement of Variables. The questionnaire was used to collect data on the independent and dependent variables. The socio-demographic and malaria-related characteristics of the respondents such as age, gender, marital status, occupation, experience of fever, and malaria were the independent variables.

The dependent variables were as follows:

(a) Health Challenges and Burden of Malaria in the Community. A multichoice question explored the three major health challenges in the community. A 5-point Likert scale question also assessed whether malaria was the most common disease in the 
community. This was scored as 1 point $=$ definitely no, 2 points $=$ probably no, 3 points $=$ unsure, 4 points = probably yes, and 5 points = definably yes

(b) Knowledge of Malaria and Malaria Prevention in Pregnancy. A total of 11 questions were used to evaluate knowledge of malaria and malaria prevention in pregnancy. Seven of the questions assessed knowledge of at-risk groups and prevention of malaria in pregnancy. They were measured on a 5 -point Likert scale scored as 1 point = definitely no, 2 points $=$ probably no, 3 points $=$ unsure, 4 points $=$ probably yes, and 5 points = definably yes. One of the questions was on self-rating of the adequacy of the knowledge of intermittent preventive therapy in pregnancy. Three multichoice questions assessed the cause of malaria, measures for malaria prevention in pregnancy, and the recommended drug for IPTp

(c) Perceived Roles and Community Ownership of Prevention of Malaria in Pregnancy. Eight questions were used to measure this variable. Seven questions assessed the roles of the community in preventing malaria in pregnancy, while one question was on the participation of the community in health programs

(d) Acceptability of Community-Directed Distribution of IPTp. Three questions measured the acceptability, importance of, and willingness to support community-directed distribution of IPTp. A single question explored the preferred route of administration (facility vs. community) of IPTp-SP among pregnant women only

Data was checked, validated, and stored in a passwordenabled computer only accessible to the primary researcher.

\subsection{Data Analysis}

2.7.1. Quantitative Data Analysis. The Statistical Package for Social Sciences (SPSS) for Microsoft Windows version 20 software [26] was used for data analysis. We calculated frequencies and proportions for categorical variables while mean and standard deviations were calculated for numeric variables.

2.7.2. Qualitative Data Analysis. Thematic analysis was used for qualitative data analysis. First, we transcribed the audio recordings from the focus group discussions and compared the transcripts with the handwritten notes in order to ensure that no important data was missed. We included some verbatim responses that reflected the original ideas of the participants. We then developed the preliminary coding framework from themes ideated from the discussion guide. The final coding framework was arrived at by comparing the transcripts with the preliminary coding framework in order to detect other themes. The data coding was done by two data coders, and disagreements were resolved using discussions and consensus. Following this, we then applied the final coding framework all the transcripts. The following themes were in the final coding framework: (a) major health problems in the community; (b) general knowledge of malaria, malaria in pregnancy, and prevention; (c) acceptability of community-directed distribution of IPTp; (d) perceived potential challenges of community-directed distribution of IPTp; and (e) sustainability of communitydirected distribution of IPTp.

2.8. Ethics Approval. Ethical approval for this study was obtained from the Research and Ethics Committees of Ebonyi State Ministry of Health and the Ebonyi State University, Ebonyi State of Nigeria. No approval number (s) was issued. Written informed consent was obtained from the respondents.

\section{Results}

3.1. Quantitative Results. The mean age of the respondents was $36.08 \pm 15.4$. The majority of the respondents were females $(73.8 \%)$, married $(75.4 \%)$, and with $49.1 \%$ having up to secondary education (Table 1).

Most of the respondents had long-lasting ITN (65.2\%) and were definitely sure of ever having had fever in the past one year $(67.1 \%)$, and over four-fifths had fever diagnosed as malaria (81.4\%). Over half of those with fever were treated with antimalarial from a health facility (58.2\%) (Table 2).

Infected mosquito bites were identified as the cause of malaria by $74.1 \%$ of the participants. Only $10.8 \%$ and $7.6 \%$ of the participants rated their knowledge of IPTp as very adequate and adequate, respectively. Most participants did not know the name of the drug used for IPTp (83.5\%). Only $7.2 \%$ and $2.1 \%$ knew that Fansidar and Amalar, respectively (common brands of sulfadoxine-pyrimethamine) were used for IPTp. Sleeping under treated mosquito treated net $(54.3 \%)$ was the most cited way of preventing malaria. Three-fourths of the respondents (75.6\%) were definitely sure that malaria was the most common disease experienced in their community (Table 3).

Over half of the respondents were definitely sure that pregnant women $(61.1 \%)$ and children (71.5\%) were at risk for malaria. The method of preventing malaria in pregnancy which most respondents definitely knew about was sleeping under long-lasting ITN (63.5\%). More than one-third (37.5\%) were definitely unaware of IPTp (Table 4 ).

Less than half of the respondents $(31.1 \%)$ were definitely convinced that the community had a role to play in preventing malaria in pregnant women and children. The respondents definitely perceived that ensuring that family members sleep under ITN (53.1\%) and sustaining efforts of projects to prevent malaria in pregnancy even after project ends (49.4\%) as community roles in preventing malaria in pregnancy (Table 5).

Majority of the participants $(60.6 \%)$ strongly agreed that community-directed distribution of IPTp-SP will improve prevention of malaria in pregnancy. About half of the respondents strongly agreed that community-directed 
TABle 1: Socio-demographic characteristics of community members in Ebonyi, Nigeria $(n=817)$.

\begin{tabular}{|c|c|c|}
\hline Variable & Frequency & Percent (\%) \\
\hline Age $($ mean $\pm S D)$ & $36.08 \pm 15.4$ & \\
\hline \multicolumn{3}{|l|}{ Gender } \\
\hline Male & 603 & 73.8 \\
\hline Female & 214 & 26.2 \\
\hline \multicolumn{3}{|l|}{ Marital status } \\
\hline Unmarried & 160 & 19.6 \\
\hline Married & 616 & 75.4 \\
\hline Divorced & 2 & 0.2 \\
\hline Separated & 5 & 0.6 \\
\hline Widowed & 34 & 4.2 \\
\hline \multicolumn{3}{|l|}{ Educational level } \\
\hline No formal education & 117 & 14.3 \\
\hline Primary education & 233 & 28.5 \\
\hline Secondary education & 401 & 49.1 \\
\hline Postsecondary education & 66 & 8.1 \\
\hline \multicolumn{3}{|l|}{ Religion } \\
\hline Christian & 802 & 98.2 \\
\hline Traditional religion & 12 & 1.5 \\
\hline Islam & 2 & 0.2 \\
\hline Others^^${ }^{\wedge}$ & 1 & 0.1 \\
\hline \multicolumn{3}{|l|}{ Employment status } \\
\hline Unemployed & 67 & 8.2 \\
\hline Paid employment & 65 & 8.0 \\
\hline Self-employment ${ }^{+}$ & 685 & 83.8 \\
\hline
\end{tabular}

$\wedge$ Others: Atheist ${ }^{+}$farmers, traders, artisans.

distribution of SP was acceptable (49.2\%) and were willing to support the project (50.8\%). Majority (74.4\%) of the pregnant respondents preferred community administration to facility administration of IPTp (Table 6).

Malaria (88.6\%), typhoid (41.6\%), and fever (39.0\%) were the three major health conditions in the community identified by the participants (Figure 1).

3.2. Qualitative Findings. One of the FGDs consisted of seven men and five women aged 24-65 years while the second FGD comprised of ten pregnant and nursing mothers aged 18-40 years.

3.3. Major (Prevalent) Health Problems in the Community. The major health challenges mentioned by the community members included malaria, fever, arthritis, peptic ulcer, convulsions in children, and eye problems in the elderly.

\subsection{Knowledge of Malaria}

3.4.1. Misconceptions about Malaria Transmission. All the participants were aware of malaria and gave the names for malaria in the local parlance. Malaria was known as isiowuwa, aru oku, and iba in the local dialect. On more probing regarding the cause of malaria, the participants gave varied opinions ranging from mosquito bites, poor personal and environmental hygienic conditions, work stress, and eating stale food.

Some of the verbatim responses are below:

"You can contact malaria through mosquito bites. Secondly drinking unclean water and through dirty hands." (Younger male).

"Eating food that is not warmed very well is another way of getting malaria because cold food gives malaria."(Older male).

3.4.2. Symptoms of Malaria. The participants readily mentioned the various symptoms of malaria, and this included headache, fever, body pains, excessive sleep, vomiting, and weakness.

3.4.3. Population Vulnerable to Malaria. All the participants identified pregnant women and children to be susceptible to malaria.

3.4.4. Effects of Malaria in Pregnancy. The discussants identified miscarriages, maternal anemia, poor growth of the foetus, and jaundice in the newborn as effects of malaria during pregnancy in addition to other symptoms of malaria which were shared by women who had experienced malaria in pregnancy.

3.4.5. Prevention of Malaria. The participants exhibited good knowledge of malaria prevention through avoidance of stagnant water and always sleeping under a net. One of the discussants highlighted the importance of sleeping under ITN and treatment for pregnant women thus:

"Pregnant woman should always sleep under the net even after delivery but when the person is infected she should be treated" (Female community member).

3.4.6. Intermittent Preventive Therapy for Malaria in Pregnancy. Only a few of the participants were aware of IPTp. Knowledge was based on direct experience via use while pregnant and indirectly through the pregnant wife experience.

This is illustrated by the comment below:

"I know about it. I got to know about it when my wife came for antenatal care. She came back with the drugs and showed it to me that they asked her to take it once. She took the drugs and there was a difference in her body" (Male community member).

3.5. Acceptability of Community-Directed Distribution of IPTp. The participants were asked to how acceptable they considered the community-directed distribution of IPT project. Before this, participants were informed that the project would involve monthly distribution of a drug designed to prevent malaria in pregnancy (and typically taken in the health facility) to pregnant women at in the community. All the participants assented that the project was acceptable and were willing to support it. One of the participants felt that community-directed distribution will ease the burden of work on health workers in health facilities. The participants were also of the opinion that this would not discourage antenatal clinic attendance. 
TABLe 2: Malaria-related characteristics of community members in Ebonyi, Nigeria $(n=817)$.

\begin{tabular}{|c|c|c|}
\hline Variable & Frequency & Percent (\%) \\
\hline \multicolumn{3}{|l|}{ Household net possession/type } \\
\hline Windows with nets & 77 & 9.4 \\
\hline Netted doors and windows & 59 & 7.2 \\
\hline Ordinary mosquito nets & 30 & 3.7 \\
\hline Long-lasting ITN & 533 & 65.2 \\
\hline None & 220 & 26.9 \\
\hline \multicolumn{3}{|l|}{ Source of ITN $(n=533)$} \\
\hline Health facility & 350 & 65.7 \\
\hline Personal purchase & 9 & 1.7 \\
\hline Friend/family member & 17 & 3.2 \\
\hline Medical outreach & 154 & 28.9 \\
\hline Others & 3 & 0.6 \\
\hline \multicolumn{3}{|l|}{ Ever had fever in the past one year } \\
\hline Definitely yes & 548 & 67.1 \\
\hline Probably yes & 43 & 5.3 \\
\hline Unsure & 1 & 0.1 \\
\hline Probably no & 21 & 2.6 \\
\hline Definitely no & 204 & 25.0 \\
\hline \multicolumn{3}{|l|}{ Was this fever diagnosed as malaria $(n=591)$} \\
\hline Yes & 481 & 81.4 \\
\hline No & 73 & 12.4 \\
\hline Unsure & 37 & 6.2 \\
\hline \multicolumn{3}{|c|}{ Where and how was the malaria diagnosed $(n=481)$} \\
\hline Health facility using microscopy & 5 & 1.0 \\
\hline Chemist using symptoms & 33 & 6.7 \\
\hline Health facility using RDT & 338 & 70.3 \\
\hline Health facility using history & 10 & 2.0 \\
\hline Chemist using RDT & 14 & 2.9 \\
\hline By family/friends & 3 & 0.6 \\
\hline Self-diagnosed using usual symptoms & 78 & 15.9 \\
\hline \multicolumn{3}{|l|}{ How was the fever treated $(n=591)$} \\
\hline Use of antimalarial from a chemist & 36 & 6.1 \\
\hline Drugs mixed form chemist & 79 & 13.4 \\
\hline Use of antimalarial from a health facility & 344 & 58.2 \\
\hline Antibiotics & 3 & 0.5 \\
\hline Paracetamol & 9 & 1.5 \\
\hline Herbal drugs/concoctions & 99 & 16.8 \\
\hline No treatment & 21 & 3.5 \\
\hline
\end{tabular}

ITN: Insecticide-Treated Net; RDT: rapid diagnostic test.

"It (community-directed IPTp-SP distribution) is good so that the nurses can have rest." (Male community member) (Chorus laughter and nodding in agreement following this comment).

"If the drugs are shared the way it's been done before (referring to Mectizan distribution), the villagers will accept it." (Female community member).

"We know every family in this community and we will inform our people to accept the drugs; after all, we are the ones benefitting from it."(Male community leader).
"I don't think it will make women stop antenatal care, they will still be coming" (Female community member).

3.6. Perceived Potential Challenges of Community Distribution of IPTp. The community members highlighted two main challenges that community distribution of IPTp could encounter. These were reduced uptake due to the fear of side effects and previous rejection of distributed drugs due to spread of false rumors. To address this, the community reinstated the need for active participation and education 
TABLE 3: General knowledge of malaria and intermittent preventive therapy in pregnancy (IPTp) among community members in Ebonyi, Nigeria $(n=817)$.

\begin{tabular}{|c|c|c|}
\hline Variable & Frequency & Percent (\%) \\
\hline \multicolumn{3}{|l|}{ Cause of malaria } \\
\hline Infected mosquito bites & 605 & 74.1 \\
\hline Too much sun & 84 & 10.3 \\
\hline Others & 76 & 9.3 \\
\hline Witchcraft & 5 & 0.6 \\
\hline Oily foods/groundnuts & 47 & 5.7 \\
\hline \multicolumn{3}{|c|}{ Is malaria the most common disease experienced in this community? } \\
\hline Definitely no & 20 & 2.4 \\
\hline Probably no & 52 & 6.4 \\
\hline Unsure & 22 & 2.7 \\
\hline Probably yes & 105 & 12.9 \\
\hline Definitely yes & 618 & 75.6 \\
\hline \multicolumn{3}{|l|}{ Ever heard of/aware of IPTp } \\
\hline Definitely no & 306 & 37.5 \\
\hline Probably no & 101 & 12.4 \\
\hline Unsure & 13 & 1.6 \\
\hline Probably yes & 125 & 15.3 \\
\hline Definitely yes & 272 & 33.3 \\
\hline \multicolumn{3}{|l|}{ Knowledge of drug used for IPTp } \\
\hline Do not know & 682 & 83.5 \\
\hline Fansidar & 59 & 7.2 \\
\hline Chloroquine & 20 & 2.4 \\
\hline Amalar & 17 & 2.1 \\
\hline Phensic & 3 & 0.4 \\
\hline Others & 36 & 4.4 \\
\hline \multicolumn{3}{|l|}{ Self-graded knowledge of IPTp } \\
\hline Grossly inadequate & 380 & 46.5 \\
\hline Inadequate & 141 & 17.3 \\
\hline Fairly adequate & 146 & 17.9 \\
\hline Adequate & 62 & 7.6 \\
\hline Very adequate & 88 & 10.7 \\
\hline \multicolumn{3}{|c|}{ Ways of preventing malaria (apart from IPTp) } \\
\hline Sleeping under treated mosquito nets & 444 & 54.3 \\
\hline Early and complete treatment & 165 & 20.2 \\
\hline Clean environment & 324 & 39.7 \\
\hline Indoor residual spraying & 142 & 17.4 \\
\hline Herbal medicines & 219 & 26.8 \\
\hline Do not know & 59 & 7.2 \\
\hline Others & 29 & 3.5 \\
\hline
\end{tabular}

IPTp: intermittent preventive therapy in pregnancy.

of community members. Some comments to highlight these are below:

"There was a time a particular drug was distributed, and the news was that when a person uses it he/she will die. Because of that, people stopped collecting drugs from strangers. So the community members should be involved so that when pregnant women see them, they will collect the drugs." (Male community leader).
"One challenge is that when people take some distributed drugs, the drug will bring out other negative effects and this can cause people to stop taking drugs." (Female community member).

3.7. Potential for Sustainability of Community Distribution of IPTp. Majority of the participants were inclined towards sustaining distribution conditional on availability of the drugs. 
TABLE 4: Knowledge of at-risk groups for malaria and prevention of malaria in pregnancy $(n=817)$.

\begin{tabular}{|c|c|c|c|c|c|c|}
\hline Variable & $\begin{array}{l}\text { Definitely } \\
\text { no }\end{array}$ & $\begin{array}{l}\text { Probably } \\
\text { no }\end{array}$ & Unsure & $\begin{array}{l}\text { Probably } \\
\text { yes }\end{array}$ & $\begin{array}{l}\text { Definitely } \\
\text { yes }\end{array}$ & Mean \pm SD \\
\hline Pregnant women are mostly affected by malaria & $46(5.6)$ & $33(4.0)$ & $\begin{array}{c}86 \\
(10.5)\end{array}$ & $\begin{array}{c}153 \\
(18.7)\end{array}$ & $499(61.1)$ & $4.26 \pm 1.1$ \\
\hline Under-five children are mostly affected by malaria & $24(2.9)$ & $15(1.8)$ & $\begin{array}{c}37 \\
(4.5)\end{array}$ & $\begin{array}{c}157 \\
(19.2)\end{array}$ & $584(71.5)$ & $4.54 \pm 0.9$ \\
\hline Sickle cell disease patients are mostly affected by malaria & $62(7.6)$ & $56(6.9)$ & $\begin{array}{c}362 \\
(44.3)\end{array}$ & $96(11.8)$ & $241(29.5)$ & $3.49 \pm 1.2$ \\
\hline $\begin{array}{l}\text { Malaria can cause serious illness and death in pregnant women and } \\
\text { children }\end{array}$ & $31(3.8)$ & $46(5.6)$ & $\begin{array}{c}57 \\
(7.0)\end{array}$ & $\begin{array}{c}191 \\
(23.4)\end{array}$ & $492(60.2)$ & $4.31 \pm 1.1$ \\
\hline $\begin{array}{l}\text { Pregnant women and their unborn children can be protected from } \\
\text { malaria by sleeping under long-lasting ITN }\end{array}$ & $41(5.0)$ & $17(2.1)$ & $\begin{array}{c}23 \\
(2.8)\end{array}$ & $\begin{array}{c}217 \\
(26.6)\end{array}$ & $519(63.5)$ & $4.41 \pm 1.0$ \\
\hline $\begin{array}{l}\text { Pregnant women and their unborn children can be protected from } \\
\text { malaria by multiple ingestion of } 3 \text { tablets of specified antimalarial } \\
\text { while pregnant }\end{array}$ & $14(1.7)$ & $30(3.7)$ & $\begin{array}{c}109 \\
(13.3)\end{array}$ & $\begin{array}{c}276 \\
(33.8)\end{array}$ & $388(47.5)$ & $4.22 \pm 0.9$ \\
\hline $\begin{array}{l}\text { Pregnant women and their unborn children can be protected from } \\
\text { malaria by early diagnosis and prompt treatment with antimalarial }\end{array}$ & $10(1.2)$ & $7(0.9)$ & $\begin{array}{c}73 \\
(8.9)\end{array}$ & $\begin{array}{c}230 \\
(28.2)\end{array}$ & $497(60.8)$ & $4.47 \pm 0.8$ \\
\hline
\end{tabular}

TABLE 5: Perceived community roles in prevention of malaria in pregnancy and childhood among community members in Ebonyi, Nigeria $(n=817)$.

\begin{tabular}{|c|c|c|c|c|c|c|}
\hline Variable & $\begin{array}{c}\text { Definitely } \\
\text { no }\end{array}$ & $\begin{array}{c}\text { Probably } \\
\text { no }\end{array}$ & Unsure & $\begin{array}{c}\text { Probably } \\
\text { yes }\end{array}$ & $\begin{array}{c}\text { Definitely } \\
\text { yes }\end{array}$ & Mean \pm SD \\
\hline $\begin{array}{l}\text { The community has a role to play in preventing malaria in her } \\
\text { pregnant women and children }\end{array}$ & $32(3.9)$ & $58(7.1)$ & $\begin{array}{c}108 \\
(13.2)\end{array}$ & $\begin{array}{c}365 \\
(44.7)\end{array}$ & $254(31.1)$ & $3.92 \pm 1.0$ \\
\hline $\begin{array}{l}\text { Community can play a role in prevention of malaria in pregnancy by } \\
\text { promoting ANC for pregnant women }\end{array}$ & $36(4.4)$ & $30(3.7)$ & $\begin{array}{c}77 \\
(9.4)\end{array}$ & $\begin{array}{c}277 \\
(33.9)\end{array}$ & $397(48.6)$ & $4.19 \pm 1.0$ \\
\hline $\begin{array}{l}\text { Community can play a role in malaria prevention in pregnancy by } \\
\text { promoting use of IPTp by pregnant women }\end{array}$ & $20(2.4)$ & $31(3.8)$ & $\begin{array}{c}107 \\
(13.1)\end{array}$ & $\begin{array}{c}286 \\
(35.0)\end{array}$ & $373(45.7)$ & $4.18 \pm 0.9$ \\
\hline $\begin{array}{l}\text { a prevention in pregnancy by } \\
\text { ITN }\end{array}$ & $22(2.7)$ & $21(2.6)$ & $\begin{array}{c}74 \\
(9.1)\end{array}$ & $\begin{array}{c}266 \\
(32.6)\end{array}$ & $434(53.1)$ & $4.31 \pm 0.9$ \\
\hline $\begin{array}{l}\text { Community can play role in malaria prevention in pregnancy by } \\
\text { referring pregnant women and children with fever to the health } \\
\text { facility }\end{array}$ & $5(0.6)$ & $26(3.2)$ & $\begin{array}{c}83 \\
(10.2)\end{array}$ & $\begin{array}{c}375 \\
(45.9)\end{array}$ & $328(40.1)$ & $4.22 \pm 0.8$ \\
\hline $\begin{array}{l}\text { Community should collaborate with partners/projects that promote } \\
\text { prevention of malaria in pregnancy }\end{array}$ & $7(0.9)$ & $22(2.7)$ & $\begin{array}{c}95 \\
(11.6)\end{array}$ & $\begin{array}{c}375 \\
(45.9)\end{array}$ & $318(38.9)$ & $4.19 \pm 0.8$ \\
\hline $\begin{array}{l}\text { Community should sustain efforts of projects to prevent malaria in } \\
\text { pregnancy even after the end of the project }\end{array}$ & $13(1.6)$ & $14(1.7)$ & $\begin{array}{c}105 \\
(12.9)\end{array}$ & $\begin{array}{c}281 \\
(34.4)\end{array}$ & $404(49.4)$ & $4.28 \pm 0.9$ \\
\hline $\begin{array}{l}\text { Health projects conducted in the community should carry the } \\
\text { community members along }\end{array}$ & $9(1.1)$ & $15(1.8)$ & $\begin{array}{c}84 \\
(10.3)\end{array}$ & $\begin{array}{c}351 \\
(43.0)\end{array}$ & $358(43.8)$ & $4.27 \pm 0$ \\
\hline
\end{tabular}

One of the participants agreed that there was a potential for sustainability of community distribution of IPTp through the use of community contributions to fund procurement of the drugs. The statement below portrays this opinion:

"If the community sees the effectiveness of the drug, they can now decide to contribute together to help bring the drug for those who need it" (Male community member).

\section{Discussion}

This study presents the baseline findings of an intervention study that implement community-directed distribution of intermittent preventive therapy for prevention of malaria in pregnancy. It explored community malaria experiences, knowledge, and perceived roles in malaria prevention in pregnancy. It also assessed the acceptability of communitydirected distribution of sulfadoxine-pyrimethamine for malaria in pregnancy.

Regarding malarial burden and experiences, a greater proportion of the respondents identified malaria as the most common health condition in the community. The prevalence of fever and diagnosis of malaria in the past one year reported by the participants was also remarkably high. This was also in consonance with the FGD findings whose participants went further to describe the local names for malaria. Although this finding was based on self-reports, it further 
TABLE 6: Acceptability of community-directed distribution of IPTp-SP among community members in Ebonyi, Nigeria.

\begin{tabular}{|c|c|c|c|c|c|c|}
\hline Parameter assessed & $\begin{array}{l}\text { Strongly } \\
\text { disagree }\end{array}$ & Disagree & Neutral & Agree & $\begin{array}{l}\text { Strongly } \\
\text { agree }\end{array}$ & Mean $\pm S D$ \\
\hline $\begin{array}{l}\text { Community-directed distribution of SP will improve prevention of } \\
\text { malaria in pregnancy }\end{array}$ & $14(1.7)$ & $26(3.2)$ & $\begin{array}{c}67 \\
(8.2)\end{array}$ & $\begin{array}{c}215 \\
(26.3)\end{array}$ & $\begin{array}{c}485 \\
(60.6)\end{array}$ & $4.41 \pm 0.9$ \\
\hline Community-directed distribution of SP is good and acceptable to me & $23(2.8)$ & $58(7.1)$ & $\begin{array}{c}97 \\
(11.9)\end{array}$ & $\begin{array}{c}237 \\
(29.0)\end{array}$ & $\begin{array}{c}402 \\
(49.2)\end{array}$ & $4.15 \pm 1.1$ \\
\hline I am willing to support community-directed distribution of SP project & $25(3.1)$ & $37(4.5)$ & $\begin{array}{c}55 \\
(6.7)\end{array}$ & $\begin{array}{c}285 \\
(34.9)\end{array}$ & $\begin{array}{c}415 \\
(50.8)\end{array}$ & $4.26 \pm 0.9$ \\
\hline \multicolumn{7}{|l|}{$\begin{array}{l}\text { Acceptability of community-directed distribution of IPTp-SP among } \\
\text { pregnant women only }(n=242)\end{array}$} \\
\hline I would rather take IPT in the community than in the health facility & $16(6.6)$ & $\begin{array}{c}32 \\
(13.2)\end{array}$ & $\begin{array}{c}14 \\
(5,8)\end{array}$ & $\begin{array}{c}52 \\
(21.5)\end{array}$ & $\begin{array}{c}128 \\
(52.9)\end{array}$ & $4.01 \pm 1.3$ \\
\hline
\end{tabular}

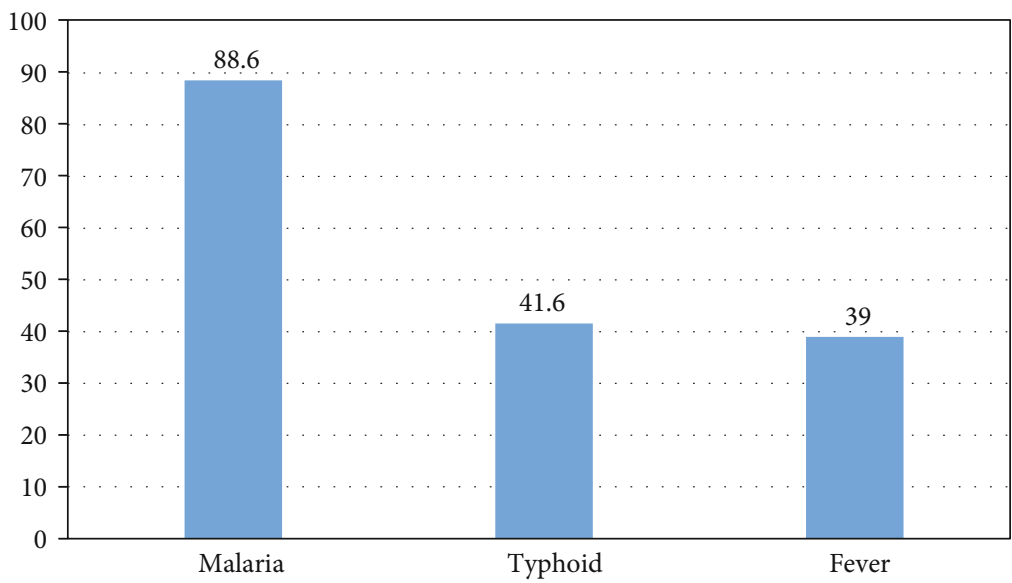

FIgURE 1: Three major health conditions in the community identified by community members in Ebonyi, Nigeria.

buttresses the already known burden and endemicity of malaria in Nigeria especially in the rural areas [7]. Other studies among rural communities have also found also that malaria was reported to be the most common disease in their settings [27-30]. Although most respondents who reportedly had malaria were treated with antimalarial drugs, the use of herbal concoctions and unidentified drugs from patent medicine venders calls for concern. Malaria care seeking from traditional healers and unqualified providers is common in the rural areas [20]. Inadequate treatment of malaria could sustain the morbidity and contribute to drug resistance especially with single/incomplete dose drug mixtures $[31,32]$. However, the authors did not ascertain whether those who received antimalarial were treated with Artemisinin-based combination therapy as globally and nationally recommended $[33,34]$.

This study revealed that most of the participants knew the cause of malaria, population groups most susceptible to malaria infection, and the effects of malaria in pregnancy. This was further corroborated by the FGD findings. The fact that malaria is endemic in Nigeria with many communitylevel control activities including awareness creation in place could account for this high knowledge levels. Also, malaria was highlighted as the most common ailment in the community, and this could also account for the knowledge levels.
Some studies have found poor knowledge of the cause of malaria among community members in Nigeria [35].

Apart from ITN use, there was suboptimal knowledge of other methods for prevention of malaria. In contrast, knowledge of malaria prevention measures has been found to be higher in other studies [35]. Sleeping under ITN was the major preventive method cited by the respondents. The use of ITN was also highlighted during the focus group discussions. The prominence of ITN could be because most respondents had ITNs. Insecticide treated mosquito net was the most known and used malaria preventive method in other community-based studies in Nigeria, Kenya, and Mozambique [8, 9, 35]. Wrong methods of prevention such as use of herbal medications for preventing malaria was mentioned by some of the respondents. Additionally, some misconceptions on the etiology of malaria such as excessive sunlight exposure, witchcraft, and food consumption (oily foods, spoilt foods) were identified from the survey and FGDs. Such misconceptions and lacunae have been reported in other studies $[36,37]$.

Majority of the participants had poor knowledge of IPTp, and this was demonstrated in both the questionbased and self-graded assessment of their knowledge of IPTp. Only few of the respondents could identify the commonly used brands of sulfadoxine-pyrimethamine used for 
IPTp, namely, Fansidar and Amalar. This finding was also supported by the FGD participants; most of whom demonstrated poor knowledge of IPTp except for pregnant women and husbands who knew that their wives had received sulfadoxine-pyrimethamine in the past. Correspondingly, another study in Southeast Nigeria found that community members could not identify Fansidar as the drug for IPTp [27]. Some possible reasons for this inadequate knowledge could be because IPTp is only taken by pregnant women with most educational activities traditionally focused on them in healthcare settings [38-41]. Likewise, studies conducted among community members have found low levels of knowledge of malaria treatment [42]. Although the wider community (men and older women inclusive) may not have been optimally engaged on IPTp in the past, they have been found to play key roles in promoting interventions aimed at reducing malaria in pregnancy [43]. Thus, it is important that community engagement and knowledge translation is integrated into programs to control and/or eliminate malaria in pregnancy. Although no additional information on IPTp was provided, most opined that IPTp would be effective in preventing malaria in pregnancy. This could be because in the study area's context, people may believe that since these drugs were manufactured by scientists and given to pregnant women (who are delicate), it should be effective. As seen from the focus group discussions and other results, the respondents knew that ITNs are protective against malaria in pregnancy. The knowledge may also influence their thinking that such drugs specially made for malaria in pregnancy should be effective as well.

Most participants affirmed that the community had a role to play in malaria prevention in pregnancy. The aspects of community roles explored include referral promoting antenatal care, promoting use of IPTp, ensuring family members sleep under ITN, referral of febrile pregnant women and children to the health facility, and collaborating with and sustaining projects on malaria prevention in pregnancy. Across all these domains, the majority of the respondents had positive perceptions towards community involvement. The felt burden of malaria in the community could have contributed to this positive role perception. Community involvement and ownership is critical to the success of community-level projects [12]. The successful implementation of the community-directed distribution of sulfadoxine-pyrimethamine project in the study community was mostly hinged on the positive attitude and cooperation shown by the community members who were actively involved in the project [19].

Social constraints such as acceptability can determine the outcome of community interventions for health problems in Africa [12]. In this study, the majority of the respondents considered the community-directed distribution of sulfadoxine-pyrimethamine acceptable and were willing to support the project. Some reasons given for the acceptability of the project by the FGD participants were previous favorable, experience with Mectizan drug distribution for neglected tropical diseases, reduction of workload in the health facility, and the beneficial effects of the project to the community. The FGD participants also expressed will- ingness to publicize the project in the community in order to promote uptake. Similar studies that evaluated community perception towards community-based programs such as community case management of malaria by community health workers also found positive community attitudes. Reduced queues in the health facilities was cited as the main reason for this disposition [18]. The high levels of acceptability closely match with the postintervention satisfaction levels with the community-directed distribution of IPTp intervention [19].

This study is not without a few limitations. Firstly, the limited number of communities involved in the survey limits the representativeness of the findings. The use of mothers recruited from health facilities could have introduced sampling bias; however, data was also collected from women of child-bearing age/mothers regardless of pregnancy status in the community. The recruitment of mothers accessing ANC/EPI services was to enhance adequate representation and inclusion of mothers who were the primary targets of the community-directed distribution. Also, the findings were based on self-reports which could be prone to social desirability bias. To mitigate confirmation bias during the FGDs, the moderator regularly summarized and clarified that responses were well captured. The moderator was careful to maintain a neutral disposition towards responses by the participants, and the researchers collated and evaluated all data equally. Since a questionnaire and discussion guide was used, question-order bias could have occurred; however, this was mitigated by pretesting, grouping of questions, and the interviewers adopted a random approach to asking the questions. Data was checked and validated and stored in a password-enabled computer only accessible to the primary researcher.

\section{Conclusions}

This study has shown that the community members surveyed recognized malaria as a prevalent disease in the community with significant symptomatic burden. There was good knowledge of malaria etiology and susceptible populations; however, knowledge of malaria prevention in pregnancy notably intermittent preventive therapy for malaria in pregnancy was suboptimal. There was positive perception of roles in malaria prevention in pregnancy and acceptability of community-directed distribution of intermittent preventive therapy for malaria in pregnancy. There was positive perception of roles in malaria prevention in pregnancy and high acceptability of community-directed distribution of IPTp. Community level malaria control programs should utilize a whole-of-community approach to optimally engage and educate the community on malaria prevention in pregnancy as well as explore community distribution approach for IPTp.

\section{Abbreviations}

FGDs: Focus group discussions

IPTp: Intermittent preventive treatment in pregnancy IPTp-SP: 
Intermittent preventive treatment with sulfadoxine-pyrimethamine

ITN: Insecticide-Treated Net

LBW: Low birth weight

MIP: $\quad$ Malaria in pregnancy

PHC: $\quad$ Primary health center

\section{Data Availability}

The datasets used and/or analyzed during the current study are available from the corresponding author on reasonable request (ijeomaninadr@gmail.com).

\section{Conflicts of Interest}

The authors declare that they do not have any conflicts of interest.

\section{Authors' Contributions}

All authors made a significant contribution to the work reported, whether that is in the conception, study design, execution, acquisition of data, analysis and interpretation, or in all these areas; took part in drafting, revising, or critically reviewing the article; gave final approval of the version to be published; have agreed on the journal to which the article has been submitted; and agreed to be accountable for all aspects of the work.

\section{Acknowledgments}

This research received financial support from the joint WHO-AFRO/TDR/EDCTP Small Grants Scheme for implementation research on infectious diseases of poverty funded (References 2019/897994 and 2019/977781).

\section{References}

[1] World Health Organization, World malaria report 2021 Geneva2021, https://www.who.int/teams/globalmalaria-programme/reports/world-malaria-report-2021.

[2] National Malaria Elimination Programme (NMEP), National Population Commission (NPopC), National Bureau of Statistics (NBS) and II, Malaria Indicator Survey 2015, NMEP, NPopC, and ICF International, Abuja, Nigeria, and Rockville, Maryland, USA, 2015.

[3] M. Fried and PE. Duffy, "Malaria during Pregnancy," Cold Spring Harb Perspect Med., vol. 7, no. 6, p. a025551, 2017.

[4] National Malaria Elimination Programme, National Malaria Strategic Plan 2014-2020, Federal Minstry of Health, Abuja Nigeria, 2014.

[5] I. N. Nglass, L. Ozor, O. Olotu, A. Momoh, and C. E. Onuekwe, "Effect of Seasonal Malaria Chemoprevention and Data Management in Health Facilities in Three Study LGAs of Adamawa State, Nigeria," Journal of Tropical Diseases, vol. 7, p. 337, 2019.

[6] National Population Commission (NPC) [Nigeria] and The DHS Program ICF Rockville, Maryland U, Nigeria Demographic and Health Survey 2018, NPC and ICF, Abuja, Nigeria and Rockville, Maryland, USA, 2019.
[7] National Malaria Elimination Programme, National Malaria Operations Research 2017-2020, Federal Mistry of Health, Abuja, Nigeria, 2017.

[8] K. W. Hlongwana, M. L. H. Mabaso, S. Kunene, D. Govender, and R. Maharaj, "Community knowledge, attitudes and practices (KAP) on malaria in Swaziland: a country earmarked for malaria elimination," Malaria Journal, vol. 8, no. 1, p. 29, 2009.

[9] P. N. Ng'ang'a, J. Mutunga, G. Oliech, and C. M. Mutero, "Community knowledge and perceptions on malaria prevention and house screening in Nyabondo, western Kenya," BMC Public Health, vol. 19, no. 1, p. 423, 2019.

[10] World Health Organization, WHO recommendations on antenatal care for a positive pregnancy experience, WHO, Geneva, 2016, http://apps.who.int/iris/bitstream/10665/250796/1/ 9789241549912-eng.pdf.

[11] Federal Ministry of Health, The 2017 Antenatal Care (ANC) Orientation Package for Health Care Providers, Federal Mistry of Health, Abuja, 2017, https://www.health.gov.ng/doc/ AnteNatal_Care_Orientation_Package_For_HealthCare_ Providers.pdf.

[12] World Health Organization on Behalf of the Special Programme for Research and Training in Tropical Diseases, Community-Directed Interventions for Major Health Problems in Africa: A Multi-Country Study: Final Report, WHO, Geneva, 2008.

[13] World Health Organization on behalf of the special programme for research and training on tropical diseases, Integrated community-based interventions, Annual Report, 2010.

[14] J. C. Okeibunor, B. C. Orji, W. Brieger et al., "Preventing malaria in pregnancy through community-directed interventions : evidence from Akwa Ibom State, Nigeria," Malaria Journal, vol. 10, p. 227, 2011.

[15] S. Gies, S. O. Coulibaly, F. T. Ouattara, C. Ky, B. J. Brabin, and U. D'Alessandro, "A community effectiveness trial of strategies promoting intermittent preventive treatment with sulphadoxine-pyrimethamine in pregnant women in rural Burkina Faso," Malaria Journal, vol. 7, no. 1, pp. 1-14, 2008.

[16] A. K. Mbonye, S. Yanow, J. Birungi, and P. Magnussen, "A new strategy and its effect on adherence to intermittent preventive treatment of malaria in pregnancy in Uganda," BMC Pregnancy and Childbirth, vol. 13, no. 1, pp. 1-7, 2013.

[17] C. Njoku, L. Nwankwo, M. Ozaki et al., "Community-wide distribution of long-lasting insecticidal nets can halt transmission of lymphatic filariasis in southeastern Nigeria," The American Journal of Tropical Medicine and Hygiene, vol. 89, no. 3, pp. 578-587, 2013.

[18] C. J. Owek, E. Oluoch, J. Wachira, B. Estambale, and Y. A. Afrane, "Community perceptions and attitudes on malaria case management and the role of community health workers," Malaria Journal, vol. 16, no. 1, p. 272, 2017.

[19] I. Okedo-Alex, I. C. Akamike, C. N. Alo et al., "Reaching the unreached : effectiveness and satisfaction with community directed distribution of sulfadoxine - pyrimethamine for preventing malaria in pregnancy in rural South-East, Nigeria," Malaria Journal, vol. 19, no. 1, p. 394, 2020.

[20] N. Orobaton, A. M. Austin, D. Abegunde et al., "Scaling - up the use of sulfadoxine - pyrimethamine for the preventive treatment of malaria in pregnancy : results and lessons on scalability, costs and programme impact from three local government areas in Sokoto State, Nigeria," Malaria Journal, vol. 15, no. 1 , p. 533, 2016. 
[21] Federal university Ndufu-alike Ikwo, About Ebonyi state, Alex Ekwueme Federal University Ndufu-Alike, Ebonyi, 2009, http://www.funai.edu.ng/about-ebonyi-state/.

[22] National Population Commission, Population and housing census of the federal republic of Nigeria, National Population Commission, Abuja, 2006, http://www.population.gov.ng/ indexphp/publications.

[23] I. A. Madu, "The structure and pattern of rurality in Nigeria," GeoJournal, vol. 75, no. 2, pp. 175-184, 2010.

[24] M. O. Araoye, Research Methodology with Statistics for Health and Social Sciences, Nathadex Publishers, Ilorin, 1st edition, 2004.

[25] S. Lwanga and S. Lemeshow, Sample size determination in health studies: a practical manual, WHO, Geneva, 1991, http://whqlibdoc.who.int/publications/9241544058_(p1-p22) .pdf.

[26] IBM Corp, IBM SPSS Statistics for Windows, Version 20.0, IBM Corp, Armonk, NY, 2011.

[27] N. G. Onyeneho, N. Idemili-Aronu, I. Igwe, and F. U. Iremeka, "Perception and attitudes towards preventives of malaria infection during pregnancy in Enugu State, Nigeria," Journal of Health, Population and Nutrition, vol. 33, no. 1, 2015.

[28] A. Das, R. K. D. Gupta, J. Friedman, M. M. Pradhan, C. C. Mohapatra, and D. Sandhibigraha, "Community perceptions on malaria and care-seeking practices in endemic Indian settings: policy implications for the malaria control programme," Malaria Journal, vol. 12, no. 1, pp. 1-12, 2013.

[29] D. Asingizwe, P. M. Poortvliet, C. J. M. Koenraadt et al., "Role of individual perceptions in the consistent use of malaria preventive measures: mixed methods evidence from rural Rwanda," Malaria Journal, vol. 18, no. 1, p. 270, 2019.

[30] B. Adhikari, K. Phommasone, T. Pongvongsa et al., "Perceptions of asymptomatic malaria infection and their implications for malaria control and elimination in Laos," PLoS One, vol. 13, no. 12, article e0208912, 2018.

[31] S. Berendes, O. Adeyemi, E. A. Oladele, O. B. Oresanya, F. Okoh, and J. J. Valadez, "Are patent medicine vendors effective agents in malaria Control? Using lot quality assurance sampling to assess quality of practice in Jigawa, Nigeria," PLoS One, vol. 7, no. 9, article e44775, 2012.

[32] B. Hanboonkunupakarn and N. J. White, "The threat of antimalarial drug resistance," Tropical Diseases, Travel Medicine and Vaccines, vol. 2, 2016.

[33] World Health Organization, Guidlines for the Treatment of Malaria, WHO, Geneva, 2015, https://apps.who.int/iris/bitstream/handle/10665/162441/9789241549127_eng.pdf.

[34] President's Malaria Initiative, National Malaria Elimination Program, Malaria Operational Plan FY 2019, Federal Mistry of Health, Abuja, 2019.

[35] R. Singh, U. V. Ebere, S. Singh, and J. Musa, "Knowledge, attitude and practices on malaria among the rural communities in Aliero, Northern Nigeria," Journal of Family Medicine and Primary Care, vol. 3, no. 1, pp. 39-44, 2014.

[36] A. Abate, A. Degarege, and B. Erko, "Community knowledge, attitude and practice about malaria in a low endemic setting of Shewa Robit Town, northeastern Ethiopia," BMC Public Health, vol. 13, no. 1, p. 1, 2013.

[37] E. Diggle, R. Asgary, G. Gore-Langton et al., "Perceptions of malaria and acceptance of rapid diagnostic tests and related treatment practises among community members and health care providers in Greater Garissa, North Eastern Province, Kenya," Malaria Journal, vol. 13, no. 1, pp. 1-12, 2014.

[38] J. Hill, K. Kayentao, M. Touré et al., "Effectiveness of antenatal clinics to deliver intermittent preventive treatment and insecticide treated nets for the control of malaria in pregnancy in Mali: a household survey," PLoS One, vol. 9, no. 3, article e92102, 2014.

[39] H. Boene, R. González, A. Valá et al., "Perceptions of malaria in pregnancy and acceptability of preventive interventions among Mozambican pregnant women: implications for effectiveness of malaria control in pregnancy," PLoS One, vol. 9, no. 2, article e86038, 2014.

[40] S. Dellicour, J. Hill, J. Bruce et al., "Effectiveness of the delivery of interventions to prevent malaria in pregnancy in Kenya," Malaria Journal, vol. 15, no. 1, p. 221, 2016.

[41] J. Hill, S. Dellicour, J. Bruce et al., "Effectiveness of antenatal clinics to deliver intermittent preventive treatment and insecticide treated nets for the control of malaria in pregnancy in Kenya," PLoS Neglected Tropical Diseases, vol. 8, no. 6, article e64913, 2013.

[42] R. K. Gupta, S. K. Raina, T. N. Shora, R. Jan, R. Sharma, and S. Hussain, "A household survey to assess community knowledge, attitude and practices on malaria in a rural population of Northern India," Journal of Family Medicine and Primary Care, vol. 5, no. 1, pp. 101-107, 2016.

[43] Z. J. L. Hildon, M. Escorcio-Ymayo, R. Zulliger et al., “"We have this, with my husband, we live in harmony": exploring the gendered decision-making matrix for malaria prevention and treatment in Nampula Province, Mozambique," Malaria Journal, vol. 19, no. 1, p. 133, 2020. 